\title{
Structural chemistry of actinide chloride complexes
}

\author{
Jennifer N. Wacker ${ }^{a}$ and Karah E. Knope ${ }^{b}$ \\ ${ }^{\text {a }}$ Georgetown University, Department of Chemistry, $37^{\text {th }}$ and O Sts. NW, Washington, DC 20057, \\ USA,jnw37@georgetown.edu \\ ${ }^{\mathrm{b}}$ Georgetown University, Department of Chemistry, $37^{\text {th }}$ and O Sts. NW, Washington, DC 20057 , \\ USA, kek44@georgetown.edu
}

Actinides play a central role in applications ranging from nuclear energy to radiotherapeutics. Motivated by these applications as well as ongoing needs to develop a predictive understanding of actinide behavior, the structural chemistry of these elements and the conditions over which various species exist and are stable has garnered increasing interest. To this end, we have examined the speciation of the early tetravalent actinides ( $\mathrm{Th}, \mathrm{U}$, and $\mathrm{Pu}$ ) in acidic aqueous media, which has particular relevance for the disposal of nuclear waste and separations efforts. Early work that probed the behavior of An(IV) metal ions in high chloride solutions with electronic absorption spectroscopy identified distinct differences between $\mathrm{Th}, \mathrm{U}$, and $\mathrm{Pu}{ }^{1}{ }^{1}$ With the development of single crystal X-ray diffraction, we now have the ability to identify the composition of those species that exist in high chloride concentrations and subsequently precipitate in the solid-state. In this work, the addition of non-coordinating N-containing heterocycles, capable of hydrogen bonding, to $\mathrm{An}(\mathrm{IV}) / \mathrm{HCl}$ solutions has resulted in the crystallization of a library of compounds. The metal ion structural units that have been isolated exhibit differences in coordination number and composition. Trends in the solid-state structural chemistry of Th, U, and Pu complexes will be discussed.

$\underline{\text { References }}$

[1] Ryan, J. L. (1961). The Journal of Physical Chemistry, 65, 1856-1859. 